\title{
Accounting for the Speed-accuracy Trade-off in Quantifying Human-in-the-loop Error Probabilities
}

\author{
Albert Ahumada ${ }^{1}$ Bettina L. Beard ${ }^{2}$ \\ NASA Ames Research Center, Moffett Field, CA, 94035 \\ Cynthia Null ${ }^{3}$ \\ NASA Langley Research Center, Hampton, VA, 23681
}

\begin{abstract}
Human-in-the loop (HITL) simulations cannot collect enough data from human operators to validate estimates of error probabilities for task components. Error rates for tasks have been estimated by using laboratory data for error rates depending on variables such as the cognitive complexity of the task. The limited channel capacity of human operators compels error rates to be strongly related to the time available for the task, the speed-accuracy trade-off. HITL simulations can provide valuable data on the time available for the operator's tasks. We propose that the response times be used in conjunction with measured speed-accuracy curves to estimate the operator error rates contributing to mission failure. Such analyses should be especially important in the estimation of error rates in offnominal situations.
\end{abstract}

\section{Nomenclature}

$a, b=$ Beta distribution parameters

$A=$ signal area

$d^{\prime}=$ signal-to-noise ratio

$E=$ signal energy

$f_{0}=$ error probability prior distribution probability density

$f_{N}=$ error probability posterior distribution probability density after $N$ trials

$F=$ cumulative distribution function

$F_{0}=$ error probability posterior cumulative distribution after $N$ trials

$F_{N}=$ error probability posterior cumulative distribution after $N$ trials

$F n=$ standard normal cumulative distribution function

$I=$ stimulus intensity level

$N=$ number of trials

$N_{0}=$ prior distribution parameter

$n_{0}=$ noise power density

$n_{c}=$ confidence level number $=1 /\left(1-p_{c}\right)$

$n_{e, 0}=$ prior distribution error probability limit number $=1 / p_{e, 0}$

$n_{e}=$ error probability number $=1 / p_{e}$

$n_{c, 0}=$ prior distribution error probability parameter number $=1 / p_{e, 0}$

\footnotetext{
${ }^{1}$ Research Psychologist, Human Systems Interface Division, NASA Ames Res. Ctr., Moffett Field, CA, 94035/Mail Stop 262-2, nonmember.

${ }^{2}$ Research Psychologist, Human Systems Interface Division, NASA Ames Res. Ctr., Moffett Field, CA, 94035/Mail Stop 262-4, nonmember.

${ }^{3}$ Technical Fellow for Human Factors, Human Systems Interface Division, NASA Ames Res. Ctr., Moffett Field, CA, 94035/Mail Stop 262-11, nonmember.
} 


$$
\begin{aligned}
& p_{c, 0}=\text { prior probability that } p_{e}<p_{e, 0} \\
& P_{d}=\text { probability of a correct discrimination } \\
& P_{I}=\text { probability of a correct identification } \\
& p_{c}=\text { confidence level as a probability } \\
& p=\text { discrete probability distribution function } \\
& p_{e}=\text { error probability } \\
& p_{e .0}=\text { prior distribution error probability limit } \\
& s=\text { sensory noise standard deviation } \\
& s_{0}=\text { sensory pattern filtered noise standard deviation } \\
& t=\text { stimulus duration } \\
& X=\text { number of trials resulting in errors }
\end{aligned}
$$

\section{Introduction}

One possible goal of HITL simulations is to provide data to evaluate the system reliability. Human operator reliability might be assessed by the frequency that errors are made in the simulation. Here we point out that even if no errors are made, the required number of opportunities for error may be impractically large if a high level of reliability must be insured. We propose using the strategy used in the perceptual sciences to ensure that stimuli can be discriminated with high reliability. Measurements are made with stimuli that lead to a measurable rate of confusion and then the low error-rate stimuli are derived from simple models. Most cognitive tasks involve a timeaccuracy trade-off, so that measurable accuracy can be achieved by making the available time short enough. Parttask simulations to measure the time-accuracy trade-off can be made more efficient through the use of sequential designs efficiently designed to estimate model parameters. Standard HITL simulations are then seen, not as a source of error rates, but as a source of distributions of times available for cognitive operations, which can then be fed into time-accuracy trade-off models to obtain error-rate estimates.

\section{The error rate measurement problem}

In the performance of critical tasks, system designers would like to have statistical evidence that the probability of human error will be less than some value $p_{e}=1 / n_{e}$ with some level of confidence $p_{c}=1-1 / n_{c}$. For example, $n_{e}$ might be 100 and $n_{c}$ might be 10 .

For values of $n_{e}>10$, it is shown in the Appendix that even if the HITL simulations opportunities resulted in no errors, the number of trials needed $\mathrm{N}$ would be

$$
N=n_{e} \ln n_{c}=2.3 n_{e} \log n_{c}
$$

For $n_{e}=100$ and $n_{c}=10$, we obtain $N=230$.

Notice that if we want the error rate to be 10 times less, $p_{e}=1 / 1000$, we need 10 times as many trials, but that if we want the confidence number to be 10 times more so that the confidence is 0.99 instead of 0.90 , we only double the number of trials needed.

The Appendix also shows that if you have the prior belief that the probability is $p_{c, 0}$ that the error rate is less than $p_{e, 0}$, the number of error-free trials $\mathrm{N}$ needed to allow the belief that it is less than $p_{e}$ with a probability of $p_{c}$, is 


$$
N=\log \left(1-p_{e}\right) / \log \left(1-p_{e}\right)-N_{0} \approx 2.3 n_{e} \log n_{c}-N_{0} .
$$

where

$$
N_{0}=\log \left(1-p_{c, 0}\right) / \log \left(1-p_{e, 0}\right) \approx 2.3 n_{e, 0} \log n_{c, 0},
$$

and $n_{e, O}$ and $n_{c, O}$ have the expected meanings, $n_{e, O}=1 / p_{e}$ and $n_{c, O}=1 /\left(1-p_{c}\right)$. The two prior assumption probabilities can be regarded as providing a number of trials that do not need to be run.

\section{Modeling Errors}

We propose that rather than measure human error rates directly, that they be estimated using models. Sensory systems can be regarded as a cascade of processes, where each level contributes its own noise. ${ }^{1}$ In the visual system at low levels, the photon noise can be dominant, but at normal working levels the neural noise from the retinal ganglion cells is regarded as the noise that limits performance. ${ }^{2-4}$ This is plausible since the information from $10^{7}$ cones must carried to the brain on $10^{6}$ nerve fibers using an inherently noisy pulse code. In the brain, resources can be devoted to reduce further degradation. A useful model for visual discrimination errors is that the visual signals are approximately linearly filtered in the retina and that spatio-temporal white noise is added by the ganglion cells. ${ }^{2}$ This signal is relayed to the cortex where an ideal statistical analysis is performed for the task required. ${ }^{5}$ The result of this analysis is a single number which has variability as a result of the noise and inherently results in sensory errors. When there are no uncertainties and the white noise is Gaussian, the ideal analysis is based on the cross correlation of the signal pattern in the domain where the noise is added with noisy signal. This results in the detectability of a signal being determined by the signal energy after the retinal filtering. ${ }^{6}$ Signal detection theory shows that the ideal observer performance is characterized by

$$
d^{\prime}=\sqrt{ }\left(E / n_{0}\right)
$$

where $E$ is the signal energy and $n_{0}$ is the noise spectral density. In vision the signal is usually regarded as a contrast signal decomposable into the product of a peak RMS contrast $C$, an energy equivalent area $A$, and energy equivalent duration $t$. The detectability $d^{\prime}$ relates to these variables as

$$
d^{\prime}=C \sqrt{ }(A t) / s_{0}
$$

where $s_{0}$ is the standard deviation of the noise resulting from the cross correlation of the noise with the signal. ${ }^{5}$

\section{Sensory errors, sensory thresholds, and JND's}

When psychologists measure sensory thresholds they assume that the trial-to-trial variations in task performance represent the effect of sensory noise. Suppose the goal is to measure the threshold for the difference in stimulus intensity at intensity level $I_{0}$. On each trial two stimuli are presented, $I_{0}$ and $I_{l}$. The probability of a correct detection can be modeled as

$$
\left.P_{d}=F n\left(I_{1}-I_{0}\right) /(s \sqrt{ } 2)\right)
$$

where $F n$ is the standard normal cumulative distribution function and $\mathrm{s}$ is the standard deviation of the sensory noise on a single presentation, $I_{0}$ or $I_{1}$. Measurements of discrimination are usually reported as the size of a justnoticeable-difference (JND) in the stimulus domain. This may be defined as the intensity difference $I_{1}-I_{0}$ that leads to $75 \%$ correct responses. Equivalently, the experiment can be regarded as measuring $\sigma$ at $\mathrm{I}_{0}$, since

$$
s=\left(I_{1}-I_{0}\right) F n^{-1}(0.75) / \sqrt{ } 2=0.477\left(I_{1}-I_{0}\right) .
$$

To ensure that sensory noise does not limit the discriminability of colors, for example, colors are often chosen to be at least $10 \mathrm{JNDs}$ apart. The probability that Gaussian noise would be large enough to cross a boundary 5 standard deviations away is about $3 \times 10^{-6}$. Notice that if we wanted to perform an experiment to ensure with $95 \%$ confidence that the error rate was less than $10^{-5}$ we would need to make over 45,000 error-free observations.

Table1 Shows distances in JNDs using the Lab color metric for colors being considered for use in control room displays. $^{7}$ 
JNDs between colors using the Lab color metric.

\begin{tabular}{|c|c|c|c|c|c|c|c|c|c|c|c|c|}
\hline \multicolumn{4}{|c|}{ RGB Input } & \multicolumn{9}{|c|}{ Normal Color Vision } \\
\hline Colors & $\mathbf{R}$ & G & B & Red & Orange & Yellow & Green & Cyan & Purple & Black & White & Gray \\
\hline Red & 255 & 0 & 0 & -- & & & & & & & & \\
\hline Orange & 255 & 160 & 80 & 42 & -- & & & & & & & \\
\hline Yellow & 255 & 255 & 0 & 79 & 44 & -- & & & & & & \\
\hline Green & 0 & 192 & 131 & 112 & 75 & 72 & -- & & & & & \\
\hline Cyan & 0 & 185 & 255 & 128 & 96 & 109 & 51 & -- & & & & \\
\hline Purple & 175 & 0 & 255 & 115 & 104 & 138 & 51 & 69 & -- & & & \\
\hline Black & 0 & 0 & 0 & 101 & 92 & 116 & 79 & 85 & 91 & -- & & \\
\hline White & 255 & 255 & 255 & 94 & 58 & 69 & 53 & 51 & 81 & 100 & -- & \\
\hline Gray & 130 & 130 & 170 & 94 & 68 & 92 & 51 & 36 & 56 & 61 & 44 & -- \\
\hline Dark Gray & 75 & 75 & 75 & 87 & 67 & 92 & 53 & 59 & 74 & 33 & 67 & 31 \\
\hline
\end{tabular}

\begin{tabular}{|c|c|c|c|c|c|c|c|c|c|c|c|c|}
\hline \multicolumn{4}{|c|}{ RGB Input } & \multicolumn{9}{|c|}{ Protanope } \\
\hline Colors & $\mathbf{R}$ & G & B & Red & Orange & Yellow & Green & Cyan & Purple & Black & White & Gray \\
\hline Red & 255 & 0 & 0 & -- & & & & & & & & \\
\hline Orange & 255 & 160 & 80 & 24 & -- & & & & & & & \\
\hline Yellow & 255 & 255 & 0 & 54 & 35 & -- & & & & & & \\
\hline Green & 0 & 192 & 131 & 31 & 21 & 54 & -- & & & & & \\
\hline Cyan & 0 & 185 & 255 & 73 & 67 & 96 & 46 & -- & & & & \\
\hline Purple & 175 & 0 & 255 & 95 & 94 & 127 & 46 & 34 & -- & & & \\
\hline Black & 0 & 0 & 0 & 60 & 80 & 113 & 72 & 83 & 82 & -- & & \\
\hline White & 255 & 255 & 255 & 65 & 47 & 66 & 34 & 37 & 71 & 100 & -- & \\
\hline Gray & 130 & 130 & 170 & 54 & 53 & 87 & 32 & 23 & 42 & 62 & 43 & -- \\
\hline Dark Gray & 75 & 75 & 75 & 39 & 53 & 88 & 40 & 53 & 63 & 33 & 67 & 30 \\
\hline
\end{tabular}

\begin{tabular}{|c|c|c|c|c|c|c|c|c|c|c|c|c|}
\hline \multicolumn{4}{|c|}{ RGB Input } & \multicolumn{9}{|c|}{ Deuteranope } \\
\hline Colors & $\mathbf{R}$ & $\mathbf{G}$ & B & Red & Orange & Yellow & Green & Cyan & Purple & Black & White & Gray \\
\hline Red & 255 & 0 & 0 & -- & & & & & & & & \\
\hline Orange & 255 & 160 & 80 & 21 & -- & & & & & & & \\
\hline Yellow & 255 & 255 & 0 & 34 & 27 & -- & & & & & & \\
\hline Green & 0 & 192 & 131 & 55 & 42 & 68 & -- & & & & & \\
\hline Cyan & 0 & 185 & 255 & 105 & 90 & 114 & 50 & -- & & & & \\
\hline Purple & 175 & 0 & 255 & 120 & 108 & 132 & 50 & 20 & -- & & & \\
\hline Black & 0 & 0 & 0 & 88 & 92 & 118 & 66 & 82 & 82 & -- & & \\
\hline White & 255 & 255 & 255 & 74 & 54 & 70 & 35 & 51 & 71 & 100 & -- & \\
\hline Gray & 130 & 130 & 170 & 80 & 68 & 94 & 26 & 27 & 40 & 61 & 45 & -- \\
\hline Dark Gray & 75 & 75 & 75 & 69 & 66 & 93 & 33 & 56 & 63 & 33 & 67 & 31 \\
\hline
\end{tabular}

The goal was to find colors that were at least 30 JNDs apart for the three color types that capture the sensitivity of the common anomolous types, deuteranomaly and protananomaly. This distance seems extreme, but the calibration experiments used stimuli with a diameter of 2 degrees of visual angle and the colored areas in the display are much smaller. If the linear dimension is reduced to only 0.2 degrees of visual angle, the JND criterion would then only be 3 JND's. One problem with trying to base the estimated sensitivity on area rather than actual ganglion cell density is that the ganglion cell density associated with the blue cones is nearly zero in the center of the fovea, leading to small field tritanopia, where the white and the yellow are not distinguishable. 
Another good example of modeling human error by sensory noise is the pattern vision model, which assumes that the visual stimulus is filtered by the optics, converted to contrast in the eye and then has spatio-temporal white noise added to it. The detection or discrimination processing is then assumed to be ideal. Once the filter properties have been measured, any experiment in pattern discrimination allows estimation of the noise level. In this model, if a stimulus is just at threshold, it can be moved a predictable distance in JNDs from threshold by increasing the area or the duration. The changing the duration changes the signal-to-noise according to the formula,

$$
d^{\prime}=d^{\prime}{ }_{0} \sqrt{\left(t / t_{0}\right),(8)}
$$

where $d^{\prime}$ is the new signal-to-noise ratio, $d_{0}^{\prime}$ is the original one, $t$ is the new duration and $t_{0}$ is the original one. If error rates are measured at short durations, this model of the human observer allows the prediction of sensory noise based error rates for longer duration stimuli, rates that are too low to be actually measured.

When observers are allowed to control their own speed, this noise model allows prediction of the speed-accuracy trade-off that results. We express the signal-to-noise ratio as

$$
d^{\prime}=d^{\prime}{ }_{1} \sqrt{t}
$$

where $d^{\prime} 1$ is the signal-to-noise ratio for a signal duration of one second. Fig. 1 shows the results from an experiment in letter identification. ${ }^{8}$

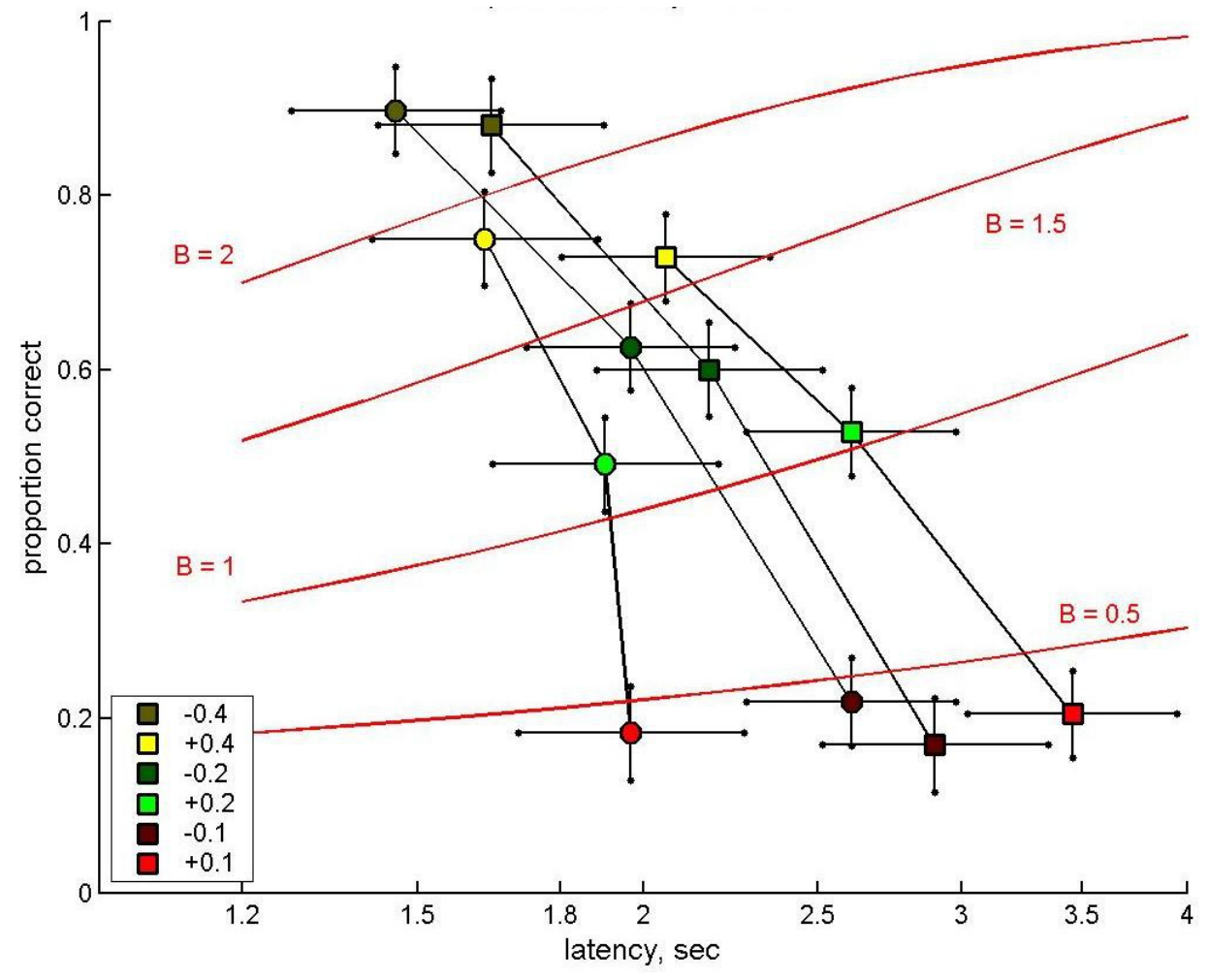

Figure 1. Letter identification accuracy vs. latency (speed-accuracy trade-off). Square symbols indicate conditions run in first group of 5 blocks. Circles indicate second group of 5 blocks. Colors indicate the contrast in percent. Error bars are $95 \%$ confidence intervals based on the observer $x$ treatment interaction. Red lines show constant performance curves for our speed-accuracy trade-off model. The parameter $\mathrm{B}$ is the parameter $d^{\prime}{ }_{1}$ in the text, the signal-to-noise ratio at a latency of one second.

Under the simplifying assumption that the differences among the 12 letters are orthogonal stimuli, a signal-to-noise ratio of $d^{\prime}$ should lead to a probability of a correct identification $P_{I}$ approximately given by

$$
P_{I}=F n\left(0.87 d^{\prime}-1.38\right)
$$


where $F n$ is the cumulative standard normal distribution and the constants are from Elliott. ${ }^{9}$ There is a striking difference between the latencies (speed) for the positive contrast conditions (light colored symbols) depending on whether they were first (squares) or second (circles). The model suggests that although the student subjects seemed to be more in a hurry to leave in the second session, they were actually performing slightly better despite the proportion of correct responses being slightly lower.

\section{Cognitive errors}

Speed-accuracy trade-off experiments in cognitive decision making have used this same basic model, assuming that the subject is accumulating noisy information over time. ${ }^{10}$

\section{Conclusion}

Human-in-the loop (HITL) simulations cannot collect enough data to validate estimates of error probabilities for task components by human operators. Error rates for tasks have been estimated by using laboratory data for error rates depending on variables such as the cognitive complexity of the task. The limited channel capacity of human operators compels error rates to be strongly related to the time available for the task, the speed-accuracy trade-off. HITL simulations can provide valuable data on the time available for the operator's tasks. We propose that the times be used in conjunction with measured or theoretical speed-accuracy curves to estimate the operator error rates contributing to mission failure. Such analyses should be especially important in the estimation of error rates in offnominal situations.

\section{Appendix}

If an experiment provides $\mathrm{N}$ independent and equally probable opportunities for an error, the probability that there will be $\mathrm{X}$ errors in $\mathrm{N}$ trials when the probability on a single trial is $p_{e}$, is given by the binomial distribution

$$
p\left(X ; N, p_{e}\right)=\left(N ! /(X !(N-X) !) p_{e}^{X}\left(1-p_{e}\right)^{N-X} .\right.
$$

The upper limit of the one sided confidence interval for $p_{e}$ with confidence level $p_{c}$ is given by the smallest value of $p_{e}$ that can be rejected by the experiment at a significance level of $1-p_{c}$. The cumulative distribution of $X, F(X ; N$, $\left.p_{e}\right)$ is the probability that no more than $X$ errors will occur,

$$
F\left(X ; N, p_{e}\right)=\sum_{x=0}^{X} p\left(x ; N, p_{e}\right)
$$

The confidence limit is thus given by the value $p_{e}$ such that

$$
F\left(X ; N, p_{e}\right)=1-p_{c} .
$$

Since the binomial distribution functions are readily available, it is straightforward to solve this equation numerically.

In the case that no errors are observed and $X=0$,

$$
F\left(0 ; N, p_{e}\right)=p\left(0 ; N, p_{e}\right)=\left(1-p_{e}\right)^{N},
$$

and the confidence limit occurs when

$$
1-p_{c}=\left(1-p_{e}\right)^{N} \text {. }
$$


Solving for $p_{e}$, we obtain

$$
p_{e}=1-\left(1-p_{c}\right)^{1 / N}
$$

If we have desired values for $p_{c}$ and $p_{e}$, we can solve for $N$

$$
N=\log \left(1-p_{c}\right) / \log \left(1-p_{e}\right) .
$$

For small $p_{e}$

$$
\log \left(1-p_{e}\right)=\ln (10) \ln \left(1-p_{e}\right) \approx-\ln (10)
$$

so

$$
N=-\ln (10) \log \left(1-p_{c}\right) / p_{e}=2.3 n_{e} \log \left(n_{c}\right),
$$

where $n_{e}=1 / p_{e}$ and $n_{c}=1 /\left(1-p_{c}\right)$.

If we want to be $99 \%$ confident $(n=100)$ that the error rate is less than $0.01\left(n_{e}=100\right)$, we find that the number of observations $N$ must be 459 by the exact formula or $2.3 \times 2 \times 100=460$, by the approximation. Note that this number is not very sensitive to the confidence level, because of the log, but gets large quickly if the error probability must be very small, i.e. if we want to know that the operator is very reliable.

With a Bayesian approach, the result is simplified if the priori distribution is chosen to be the Beta distribution. In general, if the prior distribution of $p_{e}$ is Beta with parameters $a$ and $b$, the posterior distribution after a binomial experiment with $X$ errors in $N$ trials will be Beta with parameters $a+X$ and $b+(N-X)$.

If $p_{e}$ is known to be small, it is convenient to choose prior distribution parameters $a=1$ and $b=N_{0}$, so that

$$
f_{0}\left(p_{e}\right)=N_{0}\left(1-p_{e}\right)^{N 0-1}
$$

This distribution has a mean of $1 /\left(N_{0}+1\right)$ and a standard deviation of $1 /\left(\left(N_{0}+1\right) \sqrt{ }\left(1+2 / N_{0}\right)\right.$.

The cumulative distribution is

$$
F_{0}\left(p_{e}\right)=1-\left(1-p_{e}\right)^{N 0}
$$

If our prior knowledge is that we think that the probability is $p_{c, O}$ that $p_{e}$ is less than $p_{e, O}$, then we need to choose $N_{0}$ so that

which is

$$
p_{c, 0}=1-\left(1-p_{e, 0}\right)^{N 0},(\mathrm{~A} 12)
$$

$$
N_{0}=\log \left(1-p_{c, 0}\right) / \log \left(1-p_{e, 0}\right) \approx 2.3 n_{e, 0} \log \left(n_{c, 0}\right) .
$$

When the experimental result of no errors in $N$ trials is used to update this prior, the resulting posterior distribution has the same form with $N_{0}$ replaced by $N_{0}+N$,

$$
f_{N}\left(p_{e}\right)=\left(N_{0}+N\right)\left(1-p_{e}\right)^{N 0+N} .
$$

The cumulative posterior distribution is given by

$$
F_{N}\left(p_{e}\right)=1-\left(1-p_{e}\right)^{N 0+N}
$$

The value of $N$ that ensures that the probability is $p_{c, N}$ that $p_{e}$ is less than $p_{e, N}$, satisfies

$$
N+N_{0}=\log \left(1-p_{c, N}\right) / \log \left(1-p_{e, N}\right) \approx 2.3 \mathrm{n}_{e, N} \log \left(\mathrm{n}_{c, N}\right) .
$$

American Institute of Aeronautics and Astronautics 
The quality of the approximation is shown computing the proportional error

$$
\mathrm{e}=(\ln (1-1 / N)-N) / N \text {. }
$$

For $N=11, \mathrm{e}=0.0087$; and for $N=100, \mathrm{e}=0.00010$.

\section{Acknowledgments}

This work was supported by the NASA Engineering and Safety Center.

\section{References}

1Watson, A. B. "Transfer of contrast sensitivity in linear visual networks," Visual Neuroscience, Vol. 8, pp. 65-76, 1992.

${ }^{2}$ Barton, P. G. J. "Spatio-temporal Model for the Contrast Sensitivity of the Human Eye," Dynamic Properties of Vision, Vol. VII, edited by F. Engel, H. de Ritter, F. Blommaert, Institute for Perception Research, Eindhoven, The Netherlands, 1994, pp. 3346.

${ }^{3}$ Warland, D. K., Reinagel, P., Meister, M. "Decoding visual information from a population of retinal ganglion cells." Journal of Neurophysiology, Vol. 78, No. 5, pp. 2336-2350, 1997.

${ }^{4}$ Watson, A. B., "A formula for human retinal ganglion cell receptive field density as a function of visual field location," Journal of Vision, Vol. 14, No. 7, Paper 15, 2014.

${ }^{5}$ Watson, A. B., Barlow, H. B., Robson , J. G.(1983) “What does the eye see best?” Nature , Vol. 302, No. 5907, pp. 419-422, 1983.

${ }^{6}$ Ahumada, A. J., Watson, A. B. (2013). "Visible contrast energy metrics for detection and discrimination," Human Vision and Electronic Imaging XVIII, edited by B. E. Rogowitz and T. N. Pappas., SPIE Proceedings 8651, 2013, paper 13.

7Pauli, H. "Proposed extension of the CIE recommendation on 'Uniform color spaces, color difference equations, and metric color terms,' "'Journal of the Optical Society of America, Vol. 66, No. 8, pp. 866-867, 1976.

${ }^{8}$ Ahumada, A. J., Scharff, L. F. V., "Letter Identification: Contrast Polarity and Speed-Accuracy Trade-Off Strategies," Abstracts of the Psychonomic Society 44 ${ }^{\text {th }}$ Annual Meeting, Vol. 8, 2003, p. 67.

${ }^{9}$ Elliott, P. B. “Tables of d'," Signal Detection and Recognition by Human Observers, edited by J. A. Swets,., Wiley, New York, 1964, pp. 651-684.

${ }^{10}$ Usher, M., McClelland, J. L. "The time course of perceptual choice: The leaky, competing accumulator model," Psychological Reviews, Vol. 108, No. 3, 2002, pp. 550-592. 